\title{
Producing Your Own Video Program ${ }^{1}$
}

\author{
Ricky Telg and Peyton Beattie ${ }^{2}$
}

If you're producing a video program on your own-shooting a video with your own video camera-without the help of a video production company, the first thing you need to do is to have a plan. Write a script or an outline of the content you want covered and the types of shots you'll need. Scriptwriting considerations are presented in the companion fact sheet WC024/AEC343, Producing an Educational Video, available at https://edis.ifas.ufl.edu/publication/ WC024.

Meanwhile, what about the technical side of the video production process? What equipment do you need, how do you shoot video, and what do you do about video editing? This fact sheet provides helpful hints on shooting video and producing an inexpensive video program.

\section{Video Equipment}

You should become as familiar as possible with the video camera you plan to use. Take a lot of time to practice shooting in various situations, with different lighting, visuals and composition. For a basic shoot, the minimum equipment requirements are as follows.

Video camera: Video cameras come with a wide variety of features. Most come with color viewfinders that have a small screen that extends from the video camera. Some will shoot well in dark areas with minimal lighting. All have a microphone built into the camera, but some have an audio input for an external microphone. All video cameras also have a zoom lens, which smoothly changes from a long shot (wide shot) to a close-up view without moving the camera or the object you are shooting. Video cameras shoot in one of two aspect ratios, or the width-to-height proportions of television screens. The aspect ratio for an older television screens is 4 units wide by 3 units high (4x3). For HD (high-definition or widescreen), the aspect ratio is $16 \times 9$. HD televisions are the standard sold in stores today. The $16 \times 9$ aspect ratio is the common aspect ratio for television.

Microphone: Sound may be the least thought-about component in a video shoot, but it is just as important as the visuals that are recorded. Good sound gives your program that "little extra." Microphone types include lavaliere (also known as lapel or clip-on microphones), hand-held, and shotgun. Lavaliere (pronounced LAHvuh-leer) microphones have a very limited range and are small so they are not easily seen in a video shot. They clip on to shirts and ties. Hand-held microphones are used in everyday video production, especially for television news for "person-in-the-street" interviews. Shotgun microphones are very sensitive and can pick up sound over a great distance. Shotgun microphones are usually very long and slender and are seen frequently attached to video cameras at sporting events. Keep in mind that many inexpensive consumer-grade video cameras do not have places where you can connect external microphones. Cameras with microphone inputs usually cost more, but the cost is worth it if you want the audio to sound good. If you do not have

1. This document is AEC 340, one of a series of the Department of Agricultural Education and Communication, UF/IFAS Extension. Original publication date January 1999. Revised June 2009 and July 2021. Visit the EDIS website at https://edis.ifas.ufl.edu for the currently supported version of this publication.

2. Ricky Telg, professor; Department of Agricultural Education and Communication; and Peyton Beattie, doctoral student; Department of Agricultural Education and Communication; UF/IFAS Extension, Gainesville, FL 32611.

The Institute of Food and Agricultural Sciences (IFAS) is an Equal Opportunity Institution authorized to provide research, educational information and other services

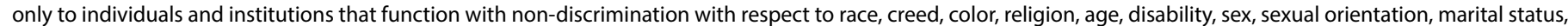

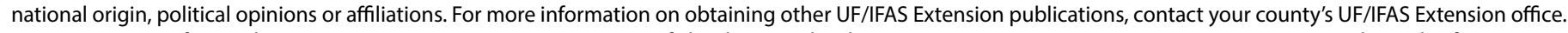
U.S. Department of Agriculture, UF/IFAS Extension Service, University of Florida, IFAS, Florida A \& M University Cooperative Extension Program, and Boards of County Commissioners Cooperating. Nick T. Place, dean for UF/IFAS Extension. 
external microphones, such as lavaliere, hand-held, or shotgun microphones, all you have to record audio with is the internal microphone on the camera. For some purposes, that microphone will be fine, but if you want to record narration or an interview, you will pick up a lot of wind noise with just an internal microphone.

A light source also needs to be taken into account. If there is not enough light, you will not be able to see a picture in your video camera's viewfinder. If the video program is to be shot outside, then you will use natural lighting. Natural lighting is any non-manmade light, such as sunlight or moonlight. In sunlight, especially, you will have to contend with harsh shadows at various times of the day and cloud cover. You may need a reflector (a large silver screen) to direct sunlight to fill in shadows on people's faces. Lighting provided by any non-natural source is called artificial lighting. If the video program is shot indoors, you likely will need portable lights, especially if you are going to shoot an interview. Regular indoor lighting, such as lighting from fluorescent bulbs, usually does not provide enough illumination for interviews. Portable light kits (a light, light stand, and a light kit case) can be purchased online through photography or video equipment websites. If you use a portable light kit, you should bring extra extension cords, as well. Lighting may also be categorized as to the strength of the light. Directional light produces a sharp beam of light, resulting in harsh shadows. Examples of directional light are direct sunlight or strong artificial lights. Diffused light refers to wide, indistinct beams of light, which produce soft shadows. Examples of diffused light are light on a cloudy day or fluorescent lamps.

If at all possible, use a tripod when interviewing a person, panning the camera (moving left and right), or tilting the camera (moving up and down). The shots will be much smoother and much less shaky. You also may wish to use a one-legged monopod for video cameras that do not weigh much. A monopod allows for easier movement of the camera, but can also compromise some of the camera's stability when shooting.

\section{Video Shooting Considerations}

The importance of using a tripod already has been mentioned. Here are some other things to consider when you're shooting video:

- Sequencing: Shoot the same shot different ways. Shoot a "long shot" (wide shot), "medium shot" (where you've zoomed in closer on the object of importance), and "close up" (where the object of importance encompasses the entire screen). This way, you draw the viewer into the video program. It also provides you with more shot choices during the editing process.

- Continuity: Have you ever watched a movie where an actor had a drink in one hand and it mysteriously switched to the other hand in the next shot? Or have you seen a television program where an actress had her arms crossed in front of her in one shot, and in the next, her arms were to her side? This is called a continuity error. To avoid this simple mistake, assign an assistant who can follow along on your video shoot to ensure that continuity is maintained. You can avoid some continuity errors by shooting cut-ins and cut-aways.

- Cut-aways: Remember to "cut away" from the action, to prevent yourself from being forced to make awkward edits in your video. For example, if you're shooting a basketball game, don't forget the audience! To transition from a shot of a basketball player on the court to another shot of the same basketball player on the court, there usually will be a "cut-away" to the fans rooting for their team.

- Cut-ins: It's the same concept as cut-aways, except instead of "cutting away" from the action (such as a shot of fans cheering at a basketball game), you "cut in" to the video. For example, if you were videotaping a person looking into a microscope, you would have a wide shot of the person staring into at the microscope. You would cut in with close-up shots of the person's hands, face, and microscope.

- Jump cuts: Avoid jump cuts whenever possible. These occur when a shot shows the same prominent person or object in different angles or different locations in backto-back shots. Cut-ins and cut-aways and shooting shots that do not contain the "prominent person or object" help minimize jump cuts.

- Shot length: One problem many amateur videographers have is recording short shots of two to five seconds in length. When they watch their footage later, they realize how little they actually recorded. Short shots are difficult to edit when you get to that stage of the production process. A good rule is to record at least 8-10 seconds per shot. You can always shorten the shots later in the video editing process.

- On-screen text: When shooting, you'll need to consider where textual material will be placed on the screen. If you're shooting an interview, remember that the bottom one-third of the screen will later contain the overlay indicating a person's name and title (called a lower-third). 
If you've zoomed in too closely, then the person's name will appear over his or her mouth or nose.

\section{- Background noise or natural sound ("nat sound")} can add "flavor" to the video, but if there's extraneous background noise that you don't want to record, you need to plan for this. For example, if you're doing a role-play with professionals in a conference room and don't want ringing telephones or hallway noise to spoil the role-play, then disconnect the telephones in the room or place signs in the hallway to let people know that videotaping is taking place. When shooting interviews outdoors, be cautious of sounds that may overpower what the person is saying. Common outdoors noises that tend to spoil video shoots include buses, loud cars, wind, and others talking.

\section{Shooting Tips}

You may wish to consider these pointers when using your video camera:

- Vary your shooting perspective. Don't shoot everything "head-on." Get above or below the object of interest.

- Establish a shot sequence of long shot (establishing shot), medium shot, and close-up.

- Don't shoot into a light source because it will make the object you record appear dark.

- If you are not shooting with a tripod, consider setting the video camera on a table so the camera doesn't shake as much.

- Unless you need continuous audio for some purpose (such as recording a marching band's song), stop the camera with the record button before catching action in another area. Stopping the camera will mean less video that has to be searched through in the editing process.

- If you are not a steady shooter and you don't have a tripod, shoot fewer close-ups. The tighter or more close-up the shot, the shakier the shot will look. Shoot wider shots or get closer to the action.

- Make pans, zooms, and tilts count. One of the ways to recognize an amateur camera operator is the number of pans (movements left and right), zooms (in and out), and tilts (up and down) that are recorded. Only use pans, zooms, and tilts when they are called for - if you're following movement or showing the size of something. It's usually best to shoot a "static" (nonmoving) shot, as well as a pan, zoom, or tilt, so you will have a couple of shots to choose from in the editing process.
- Shoot more supplemental footage (b-roll footage) than you think you will need. It is better to have more supplemental footage and not need it in the end, rather than getting to the editing process and realizing you need more supplemental footage and you do not have it. Remember, this is where sequencing is important by having different "long shots" (wide shot), "medium shots" (where you've zoomed in closer on the object of importance), and "close ups" (where the object of importance encompasses the entire screen). Sequencing gives you a variety of video shots to choose from. Having more than you need will make the video editing process smoother.

\section{Video Editing}

Editing a video is a creative process. Video editing is where you put all the various parts together into one comprehensive program. Video editing software programs digitize video, so the video can be edited in the computer, allowing you to make changes easily. It is suggested that you become very familiar with your video editing software before using it to develop a large-scale video production. Some video editing software packages are easy to learn, while some are difficult.

Video editing is also time-consuming. Video editing professionals estimate that for one minute of finished video in a program, it takes at least one hour of editing time. So for a 10-minute program, you can expect a minimum of 10 hours of editing time to complete it. Depending on the number of special effects you want to include in the video program, that amount of time may double.

Some consumer-grade video editing software programs are available for free (i.e., Adobe Premiere Rush, VideoPad, Movavi, iMovie) and function well to create video programs. Other editing programs are more expensive (i.e., Adobe Premiere, Final Cut Pro), but provide more functionality and special effects choices. These more expensive programs, with an educational discount, can run from $\$ 20-\$ 30 /$ moth. Retail prices for these pricier editing programs can be as high as $\$ 300$.

Following are some video editing tips:

- Choose supplemental footage that best tell the story. Use various shots and angles from your shot sequence (long shot, medium shot, close-up, etc.).

- Do not use everything you shoot. An educational or promotional video is not a home slide show or movie, where you show everything that you did on your family vacation. Have a purpose for what video shots you 
plan to use. This goes back to the very beginning of the production process. Know what you want the video to accomplish; then, carry that purpose all the way through the editing stage.

- Use, but do not overuse, video transitions. A video transition is the term used to indicate when an edit between shots is made. Video transitions are usually divided into the following categories: cut, dissolve or fade, and wipes.

- A cut is a direct transition from one shot to the next. It is the most commonly used transition. In video news editing, anything other than a cut is rarely used. A cut will work as a transition in about 90 percent of the time. Use cuts most of the time.

- A dissolve is a gradual change from one shot to the next. A dissolve is best used to show the passage of time or location. In most movies or television programs, you will see a dissolve used to indicate time passing.

- A similar transition to the dissolve is the fade. A fade is any shot that dissolves to black. A fade, like a dissolve, also indicates a passage of time, but is more final. For television programs, there usually is a fade at the end of each segment of the program before a commercial break starts.

- A wipe is one video picture "wiping" off and another appears. Examples of wipes are clock wipes, where the video is "wiped off" the screen with what appears to be clock hands, and checkerboard wipes. This transition style calls attention to itself and should only be used when there is a specific reason to do so.

- Properly pace the program. Pacing describes the speed or rhythm of a program, as perceived by the audience. In order to maintain audience interest and involvement, the pacing of a video program should be kept brisk. This means that most video shots should be short-in the neighborhood of five to 10 seconds in length each. However, for educational videos, the shot length could be longer. Instructional videos require that the audience be given sufficient time to learn new information.

- Remember continuity. Video editing is when you insert the cut-ins and cut-aways to cover continuity errors and jump cuts.

\section{Checklist for Producing Your Own Video Program}

- Practice with your video camera until you can operate it smoothly.

- Secure proper video equipment: video camera, tripod, lights, and a microphone.

- Shoot more supplemental footage than you think you'll need and vary the type of shots.

- Minimize pans, tilts, and zooms.

- Edit your video.

- Duplicate and distribute your video to your target audience. 